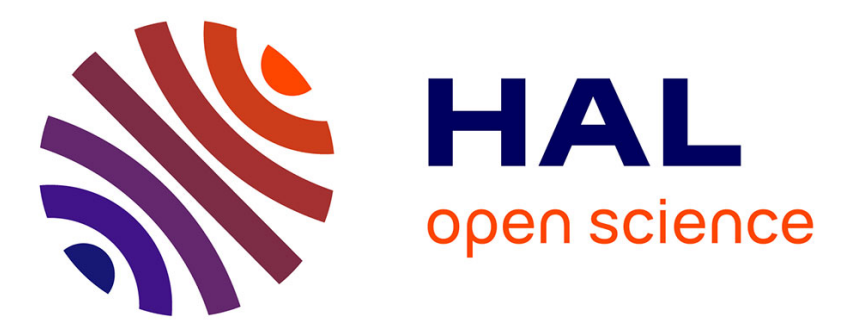

\title{
Multi-atlas synthesis for computer assisted diagnosis: Application to cardiovascular diseases
}

\author{
M. A. Zuluaga, Ninon Burgos, A. M. Taylor, S. Ourselin
}

\section{To cite this version:}

M. A. Zuluaga, Ninon Burgos, A. M. Taylor, S. Ourselin. Multi-atlas synthesis for computer assisted diagnosis: Application to cardiovascular diseases. IEEE International Symposium on Biomedical Imaging - IEEE ISBI 2015, Apr 2015, New-York, United States. pp.290-293, 10.1109/ISBI.2015.7163870 . hal-01827216

\section{HAL Id: hal-01827216 https://hal.science/hal-01827216}

Submitted on 27 Mar 2021

HAL is a multi-disciplinary open access archive for the deposit and dissemination of scientific research documents, whether they are published or not. The documents may come from teaching and research institutions in France or abroad, or from public or private research centers.
L'archive ouverte pluridisciplinaire $\mathbf{H A L}$, est destinée au dépôt et à la diffusion de documents scientifiques de niveau recherche, publiés ou non, émanant des établissements d'enseignement et de recherche français ou étrangers, des laboratoires publics ou privés. 


\title{
MULTI-ATLAS SYNTHESIS FOR COMPUTER ASSISTED DIAGNOSIS: APPLICATION TO CARDIOVASCULAR DISEASES
}

\author{
Maria A. Zuluaga ${ }^{1, \star} \quad$ Ninon Burgos ${ }^{1, \star} \quad$ Andrew M. Taylor ${ }^{2,3} \quad$ Sébastien Ourselin ${ }^{1}$ \\ ${ }^{1}$ Translational Imaging Group, Centre for Medical Image Computing, University College London, UK \\ ${ }^{2}$ Centre for Cardiovascular Imaging, UCL Institute of Cardiovascular Science, London, UK \\ ${ }^{3}$ Cardiorespiratory Division, Great Ormond Street Hospital for Children, London, UK
}

\begin{abstract}
Atlas-based analysis methods rely on the morphological similarity between the atlas and target images, and on the availability of labelled images. Problems can arise when the deformations introduced by pathologies may affect the similarity between the atlas and a patient's image. The aim of this work is to exploit the morphological dissimilarities between atlas databases and pathological images to diagnose the underlying clinical condition, while avoiding the dependence on labelled images. To this end, the proposed method is formulated under the principle of atlas-based segmentation but, instead of propagating labels, we propagate image intensities. Using a set of MR atlas databases, each database associated with a clinical condition, synthetic images are generated for a target image. The diagnosis is established by assessing the degree of similarity between the synthetic images and the target and assigning the condition of the top-ranked synthetic image. The obtained results are comparable to state-of-the-art methods using annotated images, with an accuracy of $90.0 \%$ when evaluated on a set of 60 whole heart MR images containing healthy and pathological subjects.
\end{abstract}

Index Terms - Computer-aided diagnosis, image synthesis, MR, heart

\section{INTRODUCTION}

Atlas-based segmentation is a well established method for segmenting images. It involves propagating an atlas to a target image and then applying a fusion method to combine the label images of the atlas and finally extract the structures of interest. Despite its popularity, this approach has two major drawbacks. First, to obtain a good segmentation, the morphologi-

\footnotetext{
${ }^{\star}$ Joint first authorship

This work was supported by the EPSRC (EP/H046410/1, EP/J020990/1, $\mathrm{EP} / \mathrm{K} 005278)$, the MRC (MR/J01107X/1), the EU-FP7 project VPHDARE@IT (FP7-ICT-2011-9-601055), the NIHR Biomedical Research Unit (Dementia) at UCL and the National Institute for Health Research University College London Hospitals Biomedical Research Centre (NIHR BRC UCLH/UCL High Impact Initiative), the UK National Institute for Health Research (SRF/08/01/018), and an IMPACT studentship funded jointly by Siemens and the UCL Faculty of Engineering Sciences.
}

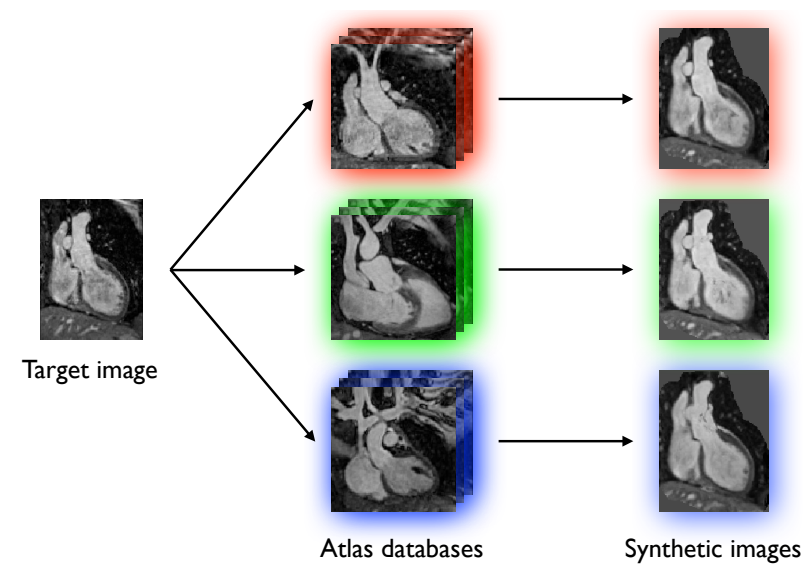

Fig. 1. MR synthesis for computer-aided diagnosis. Synthetic images are generated from the target image for each atlas database presenting a specific condition (red, green, and blue). The diagnosis corresponds to the condition of the synthetic image most similar to the target image.

cal similarity between the atlases and the target image must be sufficient. When this condition is not satisfied, the deformations induced by the morphological dissimilarities may hinder atlas registration, leading to unrepresentative segmentations. This situation is common when pathologies introduce morphological alterations in the affected organs. Second, atlasbased segmentation is strongly dependent on the availability of labelled images. In a clinical scenario or research project with limited resources, gathering a sufficient number of annotated images of pathology-specific, or even healthy subjects, is a challenge.

Different works have tried to address the problem of morphological dissimilarity. Based on the segmentation stage that they tackle, methods can be classified into four categories: atlas construction, atlas or model selection, image registration and segmentation analysis. The first class of attempts proposes to build probabilistic atlases which are flexible enough to include the variations introduced by pathologies [1]. Model 
selection techniques aim to select the atlas subset that best fits the target images as to remove potential outliers [2]. Methods addressing image registration formulate a registration framework capable of handling images containing large pathologies and large deformations [3]. Finally, segmentation analysis methods exploit the segmentation errors caused by poor atlas matching to identify the underlying pathology within the context of computer-aided diagnosis (CAD) [4]. A common characteristic of the above mentioned methods is that they do not solve the dependence on labelled images.

In this work, we follow the principle of atlas-based segmentation for CAD but, similarly to [5], instead of propagating labels, we propagate image intensities. The developed algorithm makes use of pre-acquired databases of magnetic resonance (MR) images, each database comprising subjects presenting a defined pathology. For each target image, a synthetic image is generated from each database. The diagnosis is established by comparing the synthetic images to the target.

\section{METHOD}

Following the principle that an unrepresentative atlas is more likely to lead to a poor segmentation [4], this work is based on the hypothesis that synthetic MR images generated through atlas-target matching will be more similar to the target when the patient's image and the matched database share the same clinical condition. The proposed method generates synthetic MR images through a multi-atlas information propagation scheme based on local matching of MRI features. For a given patient, we create one synthetic image for each condition using its corresponding dataset. The final diagnosis is established by assigning the condition whose synthetic image is most similar to the true image as measured by a global similarity measure (Fig. 1).

\subsection{Database preprocessing, inter-subject mapping and object location}

Given a set of clinical conditions $\Omega$, an atlas database $D_{\omega}$ is defined as the set of $N$ MR images sharing condition $\omega, \omega \in$ $\Omega$. Every image in an atlas database has an associated image mask defining a region of interest (ROI) enclosing the anatomical structure of importance. As opposed to standard atlas-based methods, these masks are not required to be highly precise. They can be, therefore, obtained through simple segmentation techniques and morphological operations (see Section 3.1).

Let $I$ be the target image to be diagnosed. MR synthesis requires the alignment of the $N$ MR images and masks from each atlas database $D_{\omega}$ to the target. This inter-subject mapping is achieved through a two-stage registration process. The images are first affinely aligned using a block matching algorithm and then non-rigidly registered using normalised mutual information. The obtained transformations are used to map the $N \mathrm{MRI} /$ mask pairs of each atlas database $D_{\omega}$ to the target image. Structure location is achieved by fusing the $N$ mapped masks of an atlas database $D_{\omega}$ through a voting scheme (voting threshold of 0.8 ). The resulting set of masks, one per condition $\omega$, is denoted $M_{\Omega}$.

\subsection{MR Synthesis}

Once mapped to the target subject, the atlas images are fused according to their morphological similarity to the target.

\subsubsection{Image/Morphological Similarity}

The morphological similarity between the target MRI and the set of registered MRIs is assessed using a local image similarity measure, the local normalised correlation coefficient (LNCC). This evaluates the quality of alignment between two images by calculating the correlation between the signals. The convolution-based LNCC, as implemented by Cachier et al. [6], between the target MR image $I$ and the $n^{t h}$ mapped image of atlas database $D_{\omega}\left(J_{n}^{\omega}\right)$, at voxel $\vec{v}$, is given by:

$$
\operatorname{LNCC}_{n, \vec{v}}=\frac{\left\langle I, J_{n}^{\omega}\right\rangle_{\vec{v}}}{\sigma(I)_{\vec{v}} \sigma\left(J_{n}^{\omega}\right)_{\vec{v}}}
$$

The means and standard deviations at voxel $\vec{v}$ are calculated using a Gaussian kernel $G_{\sigma_{G}}$ with standard deviation $\sigma_{G}$ through convolution:

$$
\begin{gathered}
\bar{I}_{\vec{v}}=\left[G_{\sigma_{G}} * I\right]_{\vec{v}} \quad \sigma(I)_{\vec{v}}=\sqrt{{\overline{I^{2}}}_{\vec{v}}-\bar{I}_{\vec{v}}^{2}} \\
\langle I, J\rangle_{\vec{v}}=\overline{I \cdot J}_{\vec{v}}-\bar{I}_{\vec{v}} \cdot \bar{J}_{\vec{v}}
\end{gathered}
$$

where $*$ denotes the convolution operator.

As suggested by Yushkevich et al. [7], registration inaccuracies are compensated using a ranking scheme where larger weight is given to the images better registered to the target image. The LNCC at each voxel is ranked across all atlas images and the ranks, noted as $R_{n \vec{v}}$, are converted to weights by applying an exponential decay function:

$$
W_{n, \vec{v}}=e^{-\beta R_{n \vec{v}}}
$$

with $W_{n \vec{v}}$ being the weight associated with the $n^{t h}$ atlas image at voxel $\vec{v}$.

\subsubsection{Intensity fusion}

Similarly to the label fusion framework developed by Cardoso et al. [2], the final synthetic MR image is obtained by a spatially varying weighted averaging process. This average is computed within a ROI $M$, defined as the intersection of each database mask:

$$
M=\bigcap_{\omega \in \Omega} M_{\omega}
$$


The fusion is restricted to the intersection to guarantee that the synthetic images generated from the different atlas databases are defined in a common region.

As the MRI intensity scale is not standardised, the images have to be normalised prior to the fusion step. We used a standard score normalisation:

$$
I_{\text {norm }}=\frac{I-\overline{I_{M}}}{\sigma\left(I_{M}\right)}
$$

where the mean and standard deviation are computed within $M$. In the following, $J_{n}^{\omega}$ refers to the normalised image.

Finally, the weights $W_{n \vec{v}}$ are used to reconstruct the synthetic MR image $I^{\omega}$ at voxel $\vec{v} \in M$ as follows:

$$
I_{\vec{v}}^{\omega}=\frac{\sum_{n=1}^{N} W_{n \vec{v}} \cdot J_{n \vec{v}}^{\omega}}{\sum_{n=1}^{N} W_{n \vec{v}}} .
$$

\subsection{Synthetic image ranking and final diagnosis}

Synthetic images are generated for each pathology represented in the databases. Each synthetic image $I^{\omega}$ is then compared to the target image $I$ using the normalised correlation coefficient (NCC). Following the idea that morphological similarity can be measured through image similarity, the synthetic images are ranked based on the NCC score. A final diagnosis is established by assigning the condition $\omega$ of the top-ranked synthetic image $I^{\omega}$ to the target image.

\section{EXPERIMENTS AND RESULTS}

In order to assess the clinical utility of the proposed CAD system, we tested its capability to diagnose dextro-transposition of the great arteries (d-TGA), a congenital heart disease in which the two major vessels that carry blood away from the heart are switched. Although the d-TGA is repaired at birth through two different procedures known as arterial switch (ASO) and atrial switch, monitoring of the patients across their life time is crucial. Identifying the anatomical variations caused by the correcting procedures from MR images is a complex task which requires high clinical expertise, and is often missed during screening.

\subsection{Data}

The dataset used for the validation was composed of $603 \mathrm{D}$, ECG- and respiratory-gated whole heart MR images acquired on a 1.5 T MR scanner (Avanto, Siemens Medical Solutions, Erlangen, Germany) at Great Ormond Street Hospital (London, UK). The images were classified by a clinical expert into three groups according to their clinical condition: ASO (20), atrial switch (20) and normal controls (20). The three groups of images were used as atlas databases $D_{\omega}, \omega \in \Omega=\{$ ASO, Atrial, Normal $\}$. Masks were obtained via Otsu thresholding, connected component analysis and morphological operations.

\begin{tabular}{|l|c|c|c|}
\hline \multirow{2}{*}{ CAD } & \multicolumn{3}{|c|}{ Real diagnosis } \\
\cline { 2 - 4 } diagnosis & ASO & Atrial & Normal \\
\hline ASO & 20 & 5 & 1 \\
Atrial & 0 & 15 & 0 \\
Normal & 0 & 0 & 19 \\
\hline
\end{tabular}

\begin{tabular}{|l|c|c|c|}
\hline \multirow{2}{*}{} & \multirow{2}{*}{ Proposed } & \multicolumn{2}{|c|}{ Segmentation-based } \\
\cline { 3 - 4 } & & Worst & Best \\
\hline Sensitivity & 100 & 87.50 & 100 \\
Specificity & 95.00 & 85.00 & 90.00 \\
Accuracy & 90.00 & 83.33 & 93.33 \\
\hline
\end{tabular}

Fig. 2. Top: CAD method confusion matrix. Bottom: Sensitivity, specificity and accuracy of the proposed method and the CAD system reported in [4].

\subsection{Results}

Validation was performed using a leave-one-out strategy, comparing the label assigned by the proposed method to the one given by the clinical expert. Figure 2 (top) summarises the results in a confusion matrix. The proposed method identified the normal condition in 19 of 20 images and ruled out the presence of a normal anatomy in 40 of 40 images. Among images flagged as pathological, the method was able to identify the correct underlying pathology in 35 of 40 images (20 of 20 for ASO and 15 of 20 for atrial). Sensitivity, specificity, and accuracy were computed and compared to an atlas segmentation-based CAD method [4] (Fig. 2, bottom). Examples of diagnosed images and the corresponding synthetic MR images are shown in Fig. 3.

In order to assess the power of NCC as the similarity measure of choice for image ranking and final diagnosis, we also analysed the NCC computed between every target image and its corresponding synthetic images. The boxplots in Fig. 4 show that NCC values are higher when the target image and the atlas database used to generate the synthetic image share the same condition.

\section{DISCUSSION AND CONCLUSIONS}

We propose a CAD method synthesising MR images using a set of pre-acquired, pathology-specific, databases, which are then compared to the target image via an image similarity measure. The diagnosis of the target image is obtained by selecting the clinical condition of the top-ranked synthetic image, i.e the synthetic image being, morphologically, the most similar to the target. The proposed CAD method has been evaluated for the classification of d-TGA congenital heart disease. Its overall system accuracy is of $90.00 \%$ in the discrimination among d-TGA variations. These results are comparable to those obtained with the segmentation-based method reported by [4]. However, our approach is more robust and 


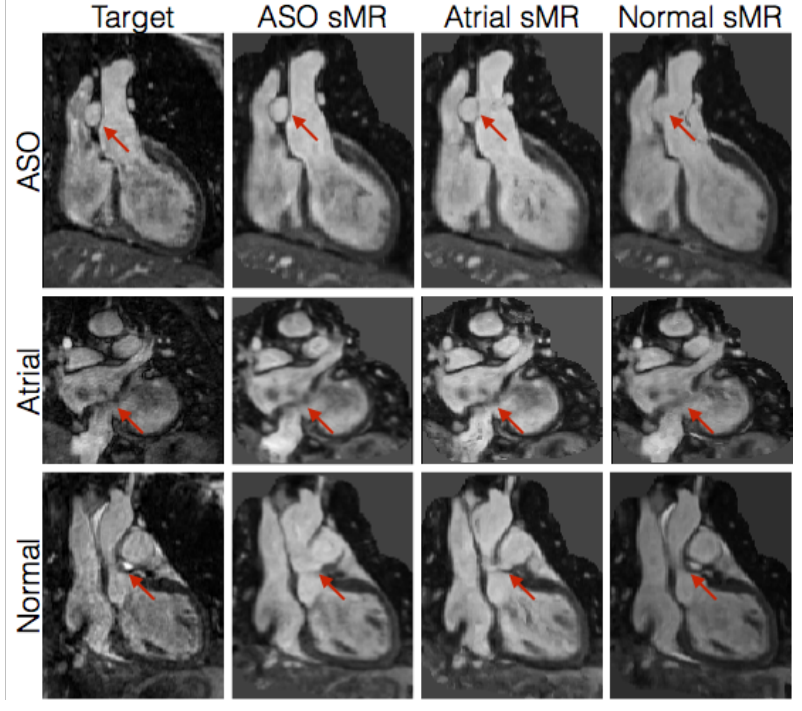

Fig. 3. For every target (first column), synthetic MR images (sMR) are obtained using three atlas databases: ASO, atrial and normal. Arrows point to areas where morphological differences between the atlas databases and the target produce anatomically inconsistent images.
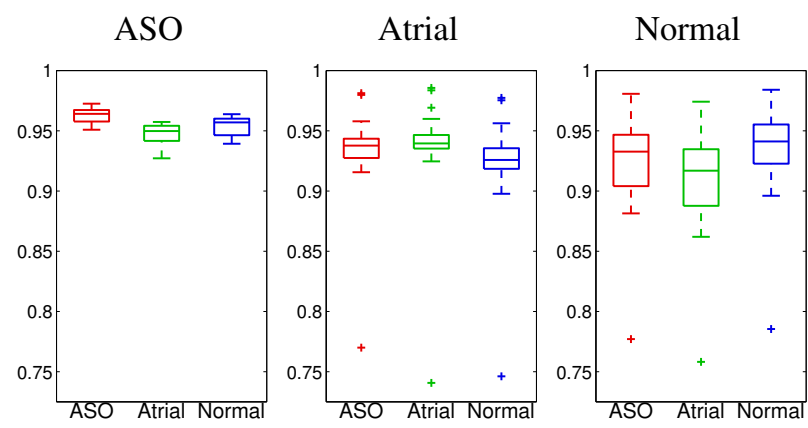

Fig. 4. Boxplots displaying the median, lower and upper quartiles, minimum and maximum of the NCC computed between the target image and the three synthetic images for the aso, atrial and normal conditions.

has the advantage of not depending on previously annotated labelled images. As can be seen in Fig. 2 (bottom), the observed performance of the CAD system in [4] could drop up to $10 \%$ depending on its configuration.

Misdiagnosed cases can be explained by the reliance on a global similarity measure to provide a final diagnosis. The global NCC score might not be able to capture morphological differences caused by the pathology if they are too subtle or it might capture differences in regions that are not relevant for the diagnosis. Strategies to overcome this limitation could include using the NCC value (as opposed to only using its rank) for the classification and adding local similarity metrics.
This paper is focused on congenital heart disease applications, but the principle can be applied for the diagnosis of pathologies in different organs. Further experiments are required to validate the method in other regions of the body.

\section{REFERENCES}

[1] M. Lorenzo-Valdes, G.I. Sanchez-Ortiz, A.G. Elkington, R.H. Mohiaddin, and D. Rueckert, "Segmentation of 4D cardiac MR images using a probabilistic atlas and the EM algorithm," Medical Image Analysis, vol. 8, pp. 255-265, 2004.

[2] M.J. Cardoso, K. Leung, M. Modat, S. Keihaninejad, D. Cash, J. Barnes, N.C. Fox, and S. Ourselin, "STEPS: Similarity and Truth Estimation for Propagated Segmentations and its application to hippocampal segmentation and brain parcelation," Medical Image Analysis, vol. 17, no. 6, pp. $671-684,2013$.

[3] X. Liu, M. Niethammer, R. Kwitt, M. McCormick, and S. Aylward, "Low-Rank to the Rescue Atlas-Based Analyses in the Presence of Pathologies," in Medical Image Computing and Computer-Assisted Intervention MICCAI 2014, 2014, vol. 8675 of LNCS, pp. 97-104.

[4] MA. Zuluaga, A.F. Mendelson, M.J. Cardoso, A.M. Taylor, and S. Ourselin, "Multi-atlas Based Pathological Stratification of D-TGA Congenital heart disease," in Biomedical Imaging (ISBI), 2014 IEEE 11th International Symposium on, April 2014, pp. 109-112.

[5] N. Burgos, M J. Cardoso, Kris Thielemans, M. Modat, S. Pedemonte, J. Dickson, A. Barnes, Rebekah Ahmed, Colin J. Mahoney, Jonathan M. Schott, J. S. Duncan, D. Atkinson, S. R. Arridge, B. F. Hutton, and S. Ourselin, "Attenuation Correction Synthesis for Hybrid PET-MR Scanners: Application to Brain Studies," IEEE Transactions on Medical Imaging, 2014.

[6] P. Cachier, E. Bardinet, D. Dormont, X. Pennec, and N. Ayache, "Iconic feature based nonrigid registration: the PASHA algorithm," Computer Vision and Image Understanding, vol. 89, no. 2-3, pp. 272-298, 2003.

[7] P.A. Yushkevich, H. Wang, J. Pluta, S.R. Das, C. Craige, B.B. Avants, M.W. Weiner, and S. Mueller, "Nearly automatic segmentation of hippocampal subfields in in vivo focal T2-weighted MRI.," NeuroImage, vol. 53, no. 4, pp. 1208-24, 2010. 